\title{
Evaluation of Movements in Tapping and Tremor from the Viewpoint of the Theory of Chaos and Self-Organization
}

\author{
Alexander A. Khadartsev ${ }^{a}$ Valeriy Matveevich Eskov ${ }^{b}$ Weidong Pan ${ }^{c}$ \\ ${ }^{a}$ Medical Institute, Tula State University, Tula, and bSurgut State University of the \\ Khanty-Mansi Autonomous Okrug-Yugra, Surgut, Russia; ' Department of Neurology, \\ Shuguang Hospital Affiliated to the Shanghai University of Traditional Chinese Medicine, \\ Shanghai, China
}

\section{Key Words}

Theory of chaos and self-organization · Motion sensors · Parkinson's disease

\section{Abstract}

Chaos in the organization of any human or animal movement has its own parameters in the form of quasi-attractors, and these parameters (quasi-attractors) have a diagnostically significant value. Motor activity of any biological entity in biomechanics consists of a real superposition of voluntary acts (i.e., having a goal and the mechanisms of its implementation) and an obligatory element of chaos when the arbitrary motor activity cannot really be implemented arbitrarily. This paper presents examples of the practical implementation of the parameters of chaos in clinical medicine and their diagnostic value.

\section{Background}

Using a new approach - the theory of chaos and self-organization (TCS) [1] - we proved the existence of continuous chaotic motion of the state vector $x=x(t)$ of any biological system (including the biomechanical) as complex of the system (complexity) [2, 3]. This indicates a basic absence of stationary modes in biosystems $(d x / d t \neq 0$ at any time) and an impossibility of any arbitrary motor activity as an inability to obtain stationary modes in the form $d x / d t=$ 0 . Even if an individual is trying to keep his or her hand with a finger fixed on a metal plate which interacts with an eddy current sensor, a stationary mode (in the form $d x / d t=0$ ) is not 
feasible for such a biological system. In this case, an arbitrary motor activity (i.e., arbitrarily keeping a finger at a particular point in space) may not be exercised arbitrarily - it is implemented chaotically, in the form $d x / d t \neq 0$, with any state of the limb being irreproducible (unrepeatable) $[4,5]$.

After 40 years of nonrecognition of the first postulate of the TCS which we have been working with all these years, and nonrecognition of synergetics as developed by H. Haken, a sensational article in the journal Nature was published by a group of scientists from Stanford University [6, 7]. There, the known statement of the founder of synergetics (H. Haken) that it makes no sense to study the individual elements of a complex system was proved $[8,9]$. The dynamic behavior of such individual elements has no informational value, and it is necessary to study the behavior of the whole system in its entirety. Today, however, there exist no formal methods of studying the dynamics of any complex system the parameters and properties of which are in continuous chaotic change [10], even if registration systems of human movement are improving [11-17].

The purpose of this study is to prove the informational significance of parameters measuring chaos in the assessment of arbitrary motor activity (tapping) to develop a new method of measuring motion parameters and to demonstrate the clinical significance of such measurements for the identification of normality and pathology.

\section{Materials and Methods}

In this paper, two types of tasks of arbitrary motor activity of the finger of a subject in the vertical direction (tapping) were used. In the first type, the finger of a test person had to touch the surface of an eddy current sensor which interacted with a plate (attached to the test person's fingertip). Practically, the subject tapped this plate on the surface of the sensor, and these movements were made with the maximum frequency of motion in the vertical direction. In the second type, the subject had not to touch the surface of the sensor, but he had to move his finger vertically with maximum frequency $[18,19]$.

These two movements differ in their degree of arbitrariness. In the first type, there is a physical limit at the lowest point (due to tapping on the sensor); in the second type, the degree of arbitrariness increases, since the subject arbitrarily restricts the movement of the finger at the highest and the lowest point. From a physical point of view, the subject has one degree of freedom, i.e., along the y-axis. However, in the first case, the movement is tightly controlled (limited) at the lowest point, and in the second case the subject arbitrarily restricts the movement of the finger at the highest and the lowest point.

Our device (fig. 1) registers the coordinate $x_{1}=y(t)$ and the coordinate $x_{2}=d y(t) / d t$ in a highly precise manner. However, into the phase plane, phase trajectories were built which were limited to a rectangle on top $\Delta S=\Delta x_{1 \max } \times \Delta x_{2 \max }$, where the segments on the axes $\mathrm{x}_{1}$ and $\mathrm{x}_{2}$ are defined as the boundaries of the intervals of measurement coordinates $\mathrm{x}_{1}$ and $\mathrm{x}_{2}$. As shown in figure 1 , the signals from the tablet (1), a surface sensor detecting coordinate $\mathrm{x}_{1}$, and the test plate (2), which is rigidly attached to the finger, are sent through an analog-to-digital converter (3) to a computer (4) equipped with software. The software calculates the second coordinate $x_{2}=d y / d t$, builds the phase plane (in the general case, there is a 6-dimensional phase space), and calculates a space of quasi-attractors ${ }^{1}$, inside which there is motion $x=$ $x(t)=\left(x_{1}, x_{2}\right)^{T}$. If three sensors (on all three axes $\mathrm{x}, \mathrm{y}$, and $\mathrm{z}$ ) are used, the device generates

1 Quasi-attractor: similar to an attractor, this is a known set of order parameters, a part of the attractor. An attractor (from English 'to attract') is a goal towards which a system is drawn. 
Fig. 1. Scheme of the biomeasuring system for the registration of tremor and tapping. Numbers are explained in the Materials and Methods section.

Khadartsev et al.: Evaluation of Movements in Tapping and Tremor from the Viewpoint of the Theory of Chaos and Self-Organization

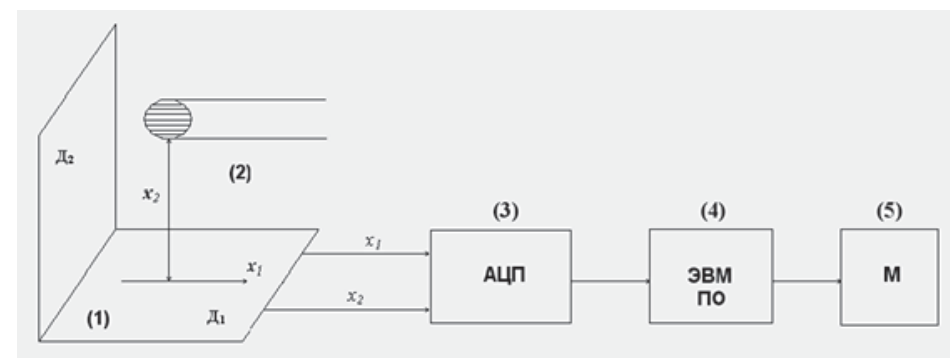

a 6-dimensional space $x=x(t)=\left(x_{1}, \ldots x_{6}\right)^{T}$ which includes all three coordinates and their velocities.

According to the principles of TCS, the limit spaces of the quasi-attractors $\Delta \mathrm{S}$ are the individual characteristics of each subject; they depend on the physiological, psychological, and physical condition of the subject. From the physical point of view, the parameters are the biological equivalent of Heisenberg's uncertainty principle, if the paired coordinates $(x$ and $d x / d t$ ) are under limiting conditions in the form

$$
\Delta x_{1} \times \Delta x_{2} \geq h / 2 \pi
$$

The analogy with quantum physics is relevant, because for any movement in biomechanics we can always introduce a measure of chaos (chaos to complexity is a basic property in TCS) in the form of parameters of quasi-attractors, and this measure will be individual for each subject.

In the Heisenberg relation, in fact, this inequation is individual for each particle; one could therefore move its mass $m$ to the right part of the inequation and write:

$$
\Delta x \times \Delta v \geq h / 2 \pi m,
$$

where $\mathrm{x}=\mathrm{a}$ coordinate and $\mathrm{v}=$ the velocity of change of this coordinate.

Thus, the analogy between quantum mechanics and TCS is based on the uncertainty of the coordinates and velocities of the particles, which are equivalent to the coordinates and velocities of the movement of any biomechanical object. In this case, inequation (2) has a pronounced individual sense (for each particle with mass $m$, we have a limit attractor uncertainty $h / 2 \pi m$ ).

Our study on tapping as a form of individual quasi-attractor was carried out in 37 healthy subjects and 15 patients with Parkinson's disease. The Ethics Committee of the Medical Institute of Tula State University approved its protocol.

\section{Results}

Firstly, any tapping, reflected in the form of mechanography and its amplitude-frequency characteristics (AFC), is unique, irreproducible, and unpredictable. During repetition of the experiment, it is possible to obtain a set of AFC which in the framework of the TCS can be represented also in the form of quasi-attractors. In this case, $\mathrm{x}_{1}$ will be the amplitude $\mathrm{A}\left(\mathrm{x}_{1}=\right.$ $A(t))$ and $\mathrm{x}_{2}$ the frequency of biomechanical movements. However, such phase trajectories are difficult to limit and to calculate (though we also already use them for diagnostics).

We give an example of registration of mechanography and the AFC (fig. 2) for a healthy subject and a patient with Parkinson's disease; it demonstrates the diagnostic value of this 'method of biological uncertainty', so we can include the identification of quasi-attractors 
Khadartsev et al.: Evaluation of Movements in Tapping and Tremor from the Viewpoint of the Theory of Chaos and Self-Organization
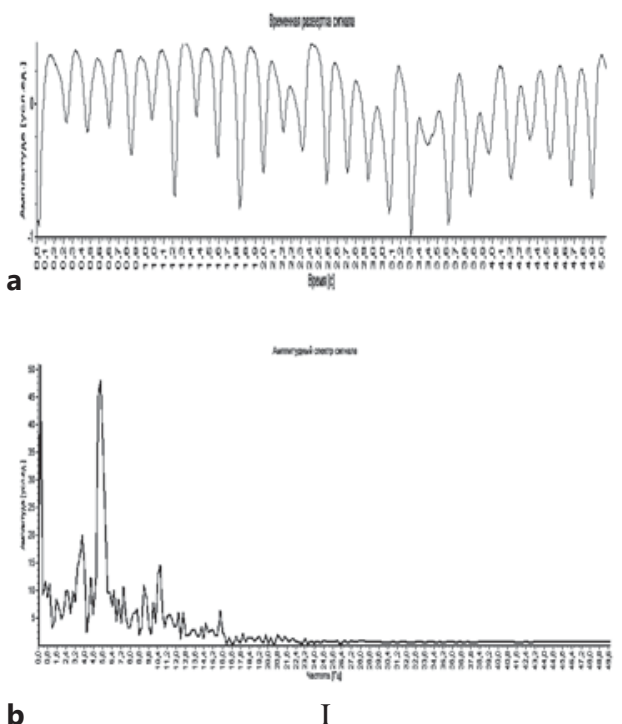

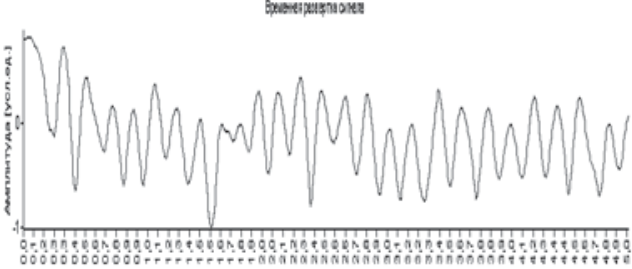

tax:

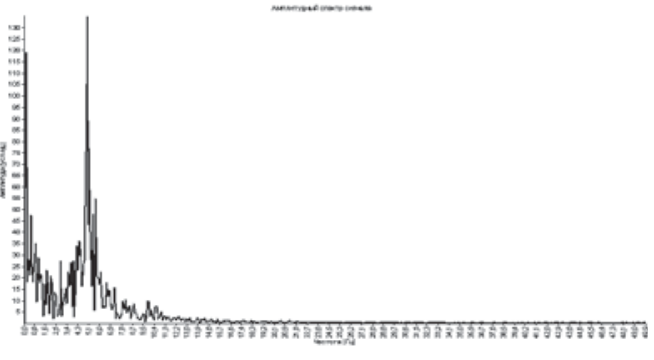

II

Fig. 2. a Mechanography of a tapping test without physical limitations. b AFC of a signal to the respective subject. I = Subject D, aged 30 years, without pathologies. II = Subject C, aged 48 years, with a diagnosis of Parkinson's disease.

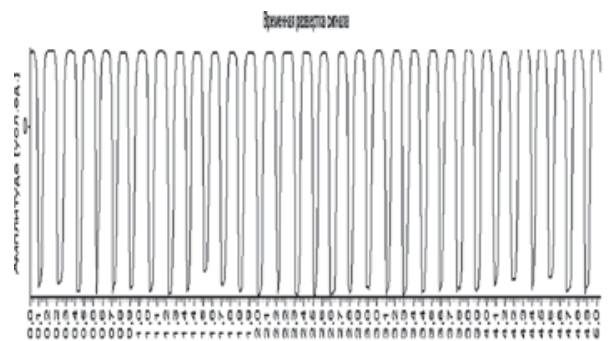

aris

$$
a^{00}
$$

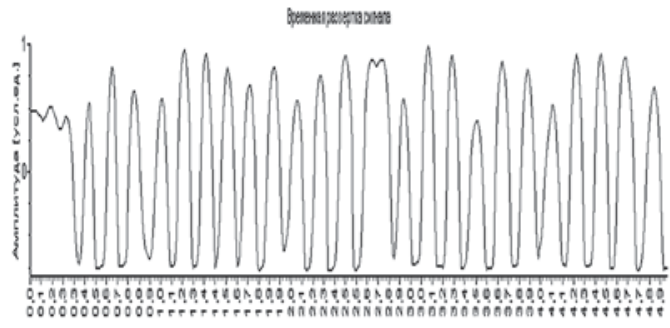

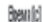

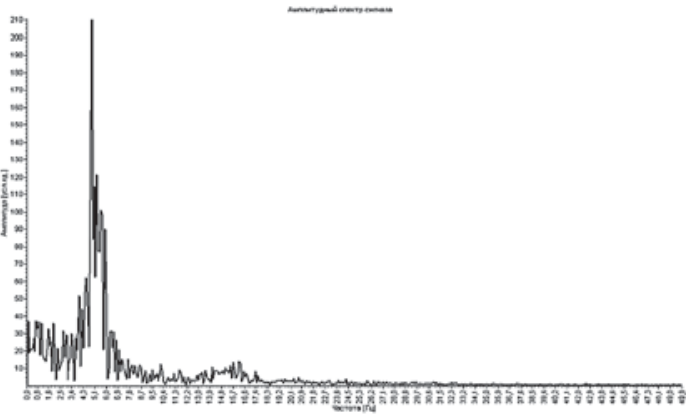

Fig. 3. a Mechanography of a tapping test in the condition of physical limitations. $\mathbf{b}$ AFC of a signal to the respective subject. I = Subject D, aged 30 years, without pathologies. II = Subject C, aged 48 years, with a diagnosis of Parkinson's disease. 
Khadartsev et al.: Evaluation of Movements in Tapping and Tremor from the Viewpoint of the Theory of Chaos and Self-Organization

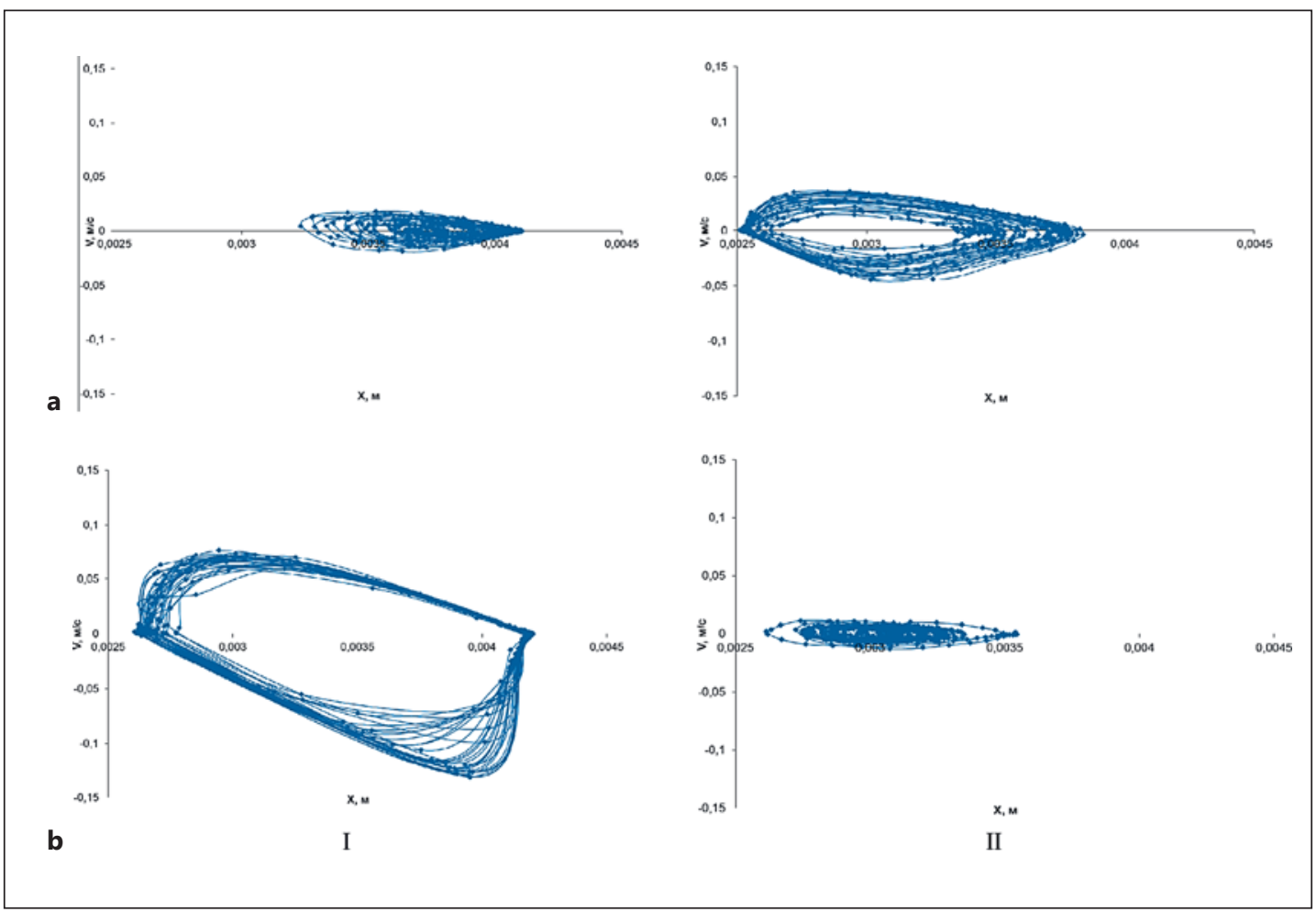

Fig. 4. a Phase plane of the vector with coordinates $x_{1}$ and $x_{2}$, where $x_{1}=x_{1}(t)$ and $x_{2}=v=d x_{1} / d t$ in a tapping test without physical limitations. $\mathbf{b}$ Phase plane of the vector with coordinates $\mathrm{x}_{1}$ and $\mathrm{x}_{2}$, where $x_{1}=x_{1}(t)$ and $x_{2}=v=d x_{1} / d t$ in a tapping test in the condition of physical limitations. I = Subject D, aged 30 years, without pathologies. II = Subject C, aged 48 years, with a diagnosis of Parkinson's disease.

(tapping) in the diagnosis of norm and pathology. In comparison, figure 3 presents the recordings of a tapping test of a healthy person and a patient with Parkinson's disease in the condition of physical limitation (i.e., touching the tablet of the sensor) and their processing.

Figures 2 and 3 demonstrate a significant difference between the signals recorded in healthy subjects and those recorded in subjects with pathology. However, this visual assessment does not provide an objective diagnostic evaluation and identification of pathology and the degree of pathology. To obtain an objective assessment of the condition of the human body, it is useful to calculate the usual and proven coordinates $x_{1}=y$ and $x_{2}=d y / d t$ (i.e., a coordinate and the velocity of a finger movement on the y-axis). In this case, the quasiattractor is easy to calculate and is very productive value (diagnostically significant). Moreover, this quasi-attractor with the space $S=\Delta x_{1} \times \Delta x_{2}$ is an important characteristic of a condition (physical or mental) of a particular person. Figure 4 presents the quasi-attractors based on the mechanography from figures 2 and 3.

$\mathrm{S}_{\mathrm{W}}$ equals the space of quasi-attractors in the tapping test without physical limitations, and $S_{F}$ equals the space of quasi-attractors in the tapping test in the condition of physical limitations. The results of calculating spaces of quasi-attractors (table 1) demonstrate that the numerical values for healthy subjects and patients with Parkinson's disease are significantly different from each other; in particular, for the healthy subject, space $S_{\mathrm{WD}}>\mathrm{S}_{\mathrm{FD}}$, whereas for the subject with Parkinson's disease there was a converse relation: $S_{\mathrm{WC}}<\mathrm{S}_{\mathrm{FC}}$. Calculating the ratio $S_{W} / S_{F}$, we obtained the following values: $S_{\mathrm{WD}} / \mathrm{S}_{\mathrm{FD}}=10.3775$ and $\mathrm{S}_{\mathrm{WC}} /$ $\mathrm{S}_{\mathrm{FC}}=0.2099$. 
Khadartsev et al.: Evaluation of Movements in Tapping and Tremor from the Viewpoint of the Theory of Chaos and Self-Organization

Table 1. Values for the spaces of quasi-attractors in a healthy subject (D) and a subject with Parkinson's disease $(\mathrm{C})$

\begin{tabular}{lll}
\hline & $\begin{array}{l}\text { Subject D } \\
\text { (healthy) }\end{array}$ & $\begin{array}{l}\text { Subject C } \\
\text { (Parkinson's disease) }\end{array}$ \\
\hline $\begin{array}{l}\text { Space } \mathrm{S}_{\mathrm{W}} \text { (tapping without physical limitations) } \\
\begin{array}{l}\text { Space } \mathrm{S}_{\mathrm{F}} \text { (test in the condition of physical } \\
\text { limitations) }\end{array}\end{array}$ & $\mathrm{S}_{\mathrm{WD}}=3.328 \times 10^{-4}$ & $\mathrm{~S}_{\mathrm{WC}}=2.26551 \times 10^{-5}$ \\
\hline
\end{tabular}

In this study (comparison of healthy subjects and subjects with Parkinson's disease), we found that for healthy subjects $S_{W} / S_{F}>1$, and for subjects with Parkinson's disease $S_{W} / S_{F}<$ 1. It is established that there are significant differences in tapping between a person without musculoskeletal pathologies and one with mobility impairments (e.g., in Parkinson's disease or essential tremor). The phase portraits of tapping in healthy persons and patients with Parkinson's disease differ in absolute and relative values.

\section{Discussion}

The proposed approache to assessing the characteristics of arbitrary motor activities showed the feasibility of using calculations of spaces of quasi-attractors in healthy individuals and patients with Parkinson's disease. These calculations have demonstrated the originality and unpredictability of tapping, which is registered in the form of mechanography and reflected by AFC. The quasi-attractor calculations suggest that quasi-attractor spaces for tapping tests of healthy subjects with no physical limitations $\left(\mathrm{S}_{\mathrm{W}}\right)$ are larger than those of healthy subjects under physical limitations $\left(\mathrm{S}_{\mathrm{F}}\right)$. In the case of Parkinson's disease, there is an inverse relationship. Thus, the ratio is $S_{W} / S_{F}>1$ for healthy subjects and $S_{W} / S_{F}<1$ for subjects with Parkinson's disease.

\section{Disclosure Statement}

The authors declare that there are no conflicts of interest regarding the publication of this article.

\section{References}

1 Eskov VM, Eskov VV, Filatova OE: Characteristic features of measurements and modeling for biosystems in phase spaces of states. Med Biol Measure 2011;53:1404.

2 Eskov VM, Khadartsev AA, Eskov VV, Gavrilenko TV, Filatov MA: Complexity is a special type of biomedical and social systems. Bull New Med Technol 2013;1:17-22.

3 Eskov VM, Khadartsev AA, Kozlova VV, Filatova OE: The use of statistical methods and multidimensional phase spaces in the evaluation of chaotic dynamics of parameters of the human neuromuscular system in terms of acoustic effects. Bull New Med Technol 2014;2:6-10.

4 Dudin NS, Rusak SN, Khadartsev AA, Khadartseva KA: New approaches in the theory of stability of biological systems - an alternative to the theory of Lyapunov. Bull New Med Technol 2011;3:336.

5 Eskov VM, Burov IV, Filatova OE, Khadartsev AA: Fundamentals of bioinformatics analysis of the dynamics of micro-chaotic behavior of biological systems. Bull New Med Technol 2012;1:15-18.

6 Jobbágy A, Harcos P, Karoly R, Fazekas G: Analysis of finger-tapping movement. J Neurosci Methods 2005;141: 29-39. 
Khadartsev et al.: Evaluation of Movements in Tapping and Tremor from the Viewpoint of the Theory of Chaos and Self-Organization

7 Churchland MM, Cunningham JP, Kaufman MT, Foster JD, Nuyujukian P, Ryu SI, Shenoy KV: Neural population dynamics during reaching. Nature 2012;487:51-58.

8 Haken H: Principles of Brain Functioning: A Synergetic Approach to Brain Activity, Behavior and Cognition. Moscow, PER SE, 2001.

9 Eskov VM, Gavrilenko TV, Kozlova VV, Filatov MA: Measurement of the dynamic parameters of microchaos in the behavior of living biosystems. Measure Tech 2012;9:1096-1101.

10 Mainzer K: Complex systems thinking: matter, mind, humanity; in Malinetsky GG (ed): A New Synthesis. Moscow, LIBROKOM, 2009.

11 Ohashi K, Nunes Amaral LA, Natelson BH, Yamamoto Y: Asymmetrical singularities in real world signals. Phys Rev E Stat Nonlin Soft Matter Phys 2003;68:65-204.

12 Pan W, Zhou H: Inclusion of integrative medicine in clinical practice. Integr Med Int 2014;1:1-4.

13 Pan W, Kwak S, Li F, Wu C, Chen Y, Yamamoto Y, Cai D: Actigraphy monitoring of symptoms in patients with Parkinson's disease. Physiol Behav 2013:119:156-160.

14 Pan W, Kwak S, Li G, Chen Y, et al: Therapeutic effect of Yang Xue Qing Nao granules on sleep dysfunction in Parkinson's disease. Chin Med 2013;8:14.

15 Pan W, Liu Y, Fang Z, Zhu X, Pan W, Kwak S, Yamamoto Y: A compound belonging to traditional Chinese medicine improves nocturnal activity in Parkinson's disease. Sleep Med 2011;12:307-308.

16 Pan W, Soma R, Kwak S, Yamamoto Y: Improvement of motor functions by noisy vestibular stimulation in central neurodegenerative disorders. J Neurol 2008;255:1657-1661.

17 Pan W, Yoshida S, Liu Q, Wu C, et al: Quantitative evaluation of severity of behavioral and psychological symptoms of dementia in patients with vascular dementia. Transl Neurodegener 2013;2:9.

18 Eskov VM, Khadartsev AA, Troitsky MS: Registration methods of different types of motion, as a basis for the development of mechanical simulators. Bull New Med Technol 2014. http://medtsu.tula.ru/VNMT/Bulletin/ E2014-1/4957.pdf.

19 Khadartsev AA, Korzhuk NL, Fudin NA, Khadartsev VA, Eskov VM, Tcherbakov DV: Mechanical simulators of the respiratory muscles in the training of the athlete. Physiotherapist 2013;2:30-39. 